\section{P174 A NOVEL NOMOGRAM TO IDENTIFY MSM AT HIGH RISK OF SYPHILIS INFECTION IN CHINA: RESULTS FROM A SERIAL CROSS-SECTIONAL STUDY}

1,2P Zhao*, ${ }^{2} \mathrm{Z}$ Yang, ${ }^{2} \mathrm{~B}$ Li, ${ }^{1} \mathrm{M}$ Xiong, ${ }^{3} \mathrm{Y}$ Zhang, ${ }^{1} \mathrm{C}$ Wang, ${ }^{2} \mathrm{Z}$ Zhou. ${ }^{1}$ Dermatology Hospital, Southern Medical University; ${ }^{2}$ School of Public Health, Southern Medical University; ${ }^{3}$ New South Wales University

\subsection{6/sextrans-2021-sti.272}

Background The purpose of this study was to develop a nomogram to identify men who have sex with men (MSM) at high risk of syphilis infection using a relatively large dataset in Southern China.

Methods A serial cross-sectional dataset of 2184 MSM from 2017 to 2019 was used to develop and validate the nomogram. All eligible MSM were randomly assigned into the training and validation datasets. Factors included in the nomogram were determined by multivariate logistic regression analysis. We used the receiver operating characteristic (ROC) curves to assess the accuracy and discriminative ability of the nomogram.

Results A total of 2184 MSM were recruited in this study. The overall syphilis prevalence was $18.1 \%$ (396/2184). The multivariate logistic regression found that MSM who were older age, sought sexual partners through non-internet route, had not consistently used condom in the last 6 months, had commercial sex with men in the last 6 months and had infected STD in the past year were more likely infected with syphilis.

We generated the nomogram to identify MSM at high risk of syphilis infection in the training cohort (C-index 0.80 [95\% CI $0.76-0.84$ ], sensitivity $86.9 \%$, specificity $64.0 \%$ ); in validation cohort $(0.79[0.75-0.84], 86.0 \%, 63.8 \%)$. When the total score is $>46.5$, MSM should be considered at high risk of syphilis infection.

Conclusions A nomogram for early risk assessment of syphilis infection among MSM has been developed and validated, which is easy to use and identifies MSM who are at higher risk. The proposed nomogram shows good assessment performance.

\section{P175 WHEN RESISTANCE TESTS FAIL: DNA NEGATIVE MYCOPLASMA GENITALIUM DETECTED BY RNA ASSAYS}

H Coleman*, E Wallis, N Essaji, C Fairhead, A Nori. Guy's and St. Thomas' Hospital, London, UK

\subsection{6/sextrans-2021-sti.273}

The clinical significance DNA negative Mycoplasma genitalium (MG) detected by RNA assay is unknown. Macrolide resistance rates in the UK are high. When using resistance guided therapy, in the absence of a resistance result, empirical therapy of DNA negative MG is usually Moxifloxacin based therapy.

A retrospective case notes review for all patients tested for MG between January-December 2019 at a large London sexual health service was carried out. Hologic ${ }^{\mathrm{TM}}$ Aptima ${ }^{\circledR}$ transcription medicated amplification (TMA) MG positive samples were confirmed at Public Health England an in-house multiplex real-time PCR incorporating 2 targets ( $\mathrm{MgPa}$ and gap). DNA negative samples were identified. Information on demographics, rationale for testing, treatment and outcome was collected.
2346 patients were tested for MG during the period. 536/ $2346(23 \%)$ patients tested RNA positive. 125/536 (23\%) were macrolide sensitive, 227/536 (42\%) were macrolide resistant. Insufficient DNA for resistance test was reported in 184/536 (34\%). 114/184 (62\%) were DNA negative on two targets.

The majority of DNA negative samples were urine $(81 / 114$, $71 \%)$; from heterosexuals $(92 / 114,81 \%)$; who were symptomatic (76/114, 67\%). 29/114 (25\%) did not have a valid indication for testing. The majority of patients $(71 / 114,62 \%)$ had Moxifloxacin based therapy of whom 45/71 (63\%) had a negative test of cure (TOC) by TMA. 25/71 (35\%) had no TOC, $1 / 71(1 \%)$ had a persistent DNA negative result.

When using RNA assays for MG testing, DNA negative MG is common and may represent cleared infection, cross reactivity with other Mycoplasma spp., or clinically insignificant/non-pathogenic carriage and the risks outweigh the benefits for treatment with Moxifloxacin. Clinico-pathological outcome data on DNA negative $\mathrm{MG}$ is required to inform interpretation of these results.

\section{P179 ACCESS TO, AND USAGE OF, ONLINE POSTAL SEXUALLY TRANSMITTED INFECTION SELF-SAMPLING SERVICES: A SCOPING REVIEW}

K Sumray, K Lloyd*, J Gibbs. Institute for Global Health, University College London, London, UK

\subsection{6/sextrans-2021-sti.274}

Background Increasing service user demand and rising incidence of key sexually transmitted infections (STIs), alongside developments in diagnostics and digital health provision, at a time when services have faced fragmentation and reduction in funding, have resulted in a huge expansion in online postal sexually transmitted infection (STI) selfsampling services across some parts of the UK. This review aimed to explore who is accessing and using online postal self-sampling services in the UK, the acceptability of these services and whether they are exacerbating sexual health inequalities.

Methods Following the PICO (Population Intervention Comparators Outcome) framework, a systematic search was conducted in nine databases in June 2020. Included studies were published between 01/2010-06/2020, in the English language, based on pre-agreed inclusion/exclusion criteria. A second reviewer carried out abstract and full text screening. 15 studies were included. Extracted data were analysed using descriptive statistics and thematic analysis.

Results Study designs were heterogeneous, including quantitative, qualitative and mixed method analysis, and were therefore appraised using the Mixed Methods Appraisal Tool. Overall, studies were of good quality. However, the majority were either evaluating a single site/testing provider, were exploratory or observational. Few studies collected comprehensive demographic data. Individuals accessing online postal STI self-sampling services tended to be asymptomatic, white, women, over 20s, and from less deprived areas. These services tended to increase overall STI testing demand and access. There were varied results on whether services reduced time to treatment. Services were deemed highly acceptable if they were trustworthy, reliable, convenient, and improved patient choice. 
Conclusion Existing and new services must complete comprehensive evaluations to establish whether services are inclusive, accessible and address, rather than exacerbate, sexual health inequalities. Now is a critical time to identify the effectiveness of these digital health interventions given the wave of service expansions across the UK.

\section{P180 SELF-SAMPLING DEMONSTRATES COMPARABLE SENSITIVITY AND SPECIFICITY TO CLINICIAN-SAMPLING FOR HPV TESTING AMONG MSM IN CHINA}

${ }^{1} \mathrm{Y} \mathrm{Ni}{ }^{*},{ }^{1} \mathrm{Y} \mathrm{Lu},{ }^{2} \mathrm{X} \mathrm{He},{ }^{2} \mathrm{Y} \mathrm{Li},{ }^{1} \mathrm{C} \mathrm{Xu},{ }^{1} \mathrm{~W}$ Tang. ${ }^{1}$ University of North Carolina At Chapel Hill Project-China, Guangzhou, China; ${ }^{2}$ Zhuhai Xutong Voluntary Services Center, Zhuhai, China

10.1136/sextrans-2021-sti.275

Human papillomavirus (HPV) can cause genital warts and cervical, anal, and penile cancers. HPV self-sampling is an acceptable and feasible approach for HPV testing among women. Despite emerging research stated a higher risk of HPV infection among men who have sex with men (MSM), few guidelines are available for HPV self-sampling among MSM. Adding HPV self-sampling as a complement may benefit MSM and improve STI testing services among key populations. This study aimed to evaluate the feasibility and accuracy of HPV self-sampling among MSM.

MSM who were aged 18 or above, had sex with men in the past year were recruited. Eligible participants followed the instruction to self-collect specimens using oral fluid, penile, and rectal swab. Then a clinician or trained staff collected specimens from same areas. All specimens were processed using PCR test for 14 high-risk subtypes and 2 low-risk subtypes. PCR results were defined as the gold standard when assessing the performance of self-sampling and clinician-sampling. Sensitivity and specificity were calculated for each approach independently, and then chi-square test was used to compare two approaches.

A total of 211 MSM were recruited and tested from April to October 2020 in Zhuhai, China. The mean age of MSM was 31 years old $(\mathrm{SD}=7.9)$. The overall prevalence of HPV among participants was 49\% (103/211). Clinician-sampled specimens detected 91 of 103 MSM infected with HPV, with a sensitivity of $88.3 \%$ (95\% CI: 80.2-93.6), and specificity of $100.0 \% \quad$ (95\% CI: 95.7-100.0), respectively. Self-sampled specimens detected 81 of 103 MSM infected with HPV, with a sensitivity of $78.6 \%$ (95\% CI: 69.2-85.9), and a specificity of $100.0 \%$ (95\% CI: 95.7-100.0), respectively. The sensitivity was comparable between the clinician-sampling and self-sampling among MSM $(\mathrm{P}=0.09)$.

HPV self-sampling is feasible among MSM and it holds the potential in scaling-up HPV testing services among key populations.

\section{P181 PUBLIC HEALTH DECISION-MAKERS' PERSPECTIVES ON APPROACHES TO ECONOMIC EVALUATION FOR SEXUALLY TRANSMITTED INFECTION CONTROL PROGRAMMES}

'S Bloch*, 'L Jackson, 'E Frew, ${ }^{2} \mathrm{~J}$ Ross. 'University of Birmingham, Birmingham, UK; ${ }^{2}$ Whittall Street Clinic, University Hospitals Birmingham NHS Foundation Trust, Birmingham, UK

10.1136/sextrans-2021-sti.276
Background Economic evaluations aim to inform decision-makers about the cost-effectiveness of health interventions. However, currently economic evaluations may be underutilised by local public health decision-makers.

Whilst research has been conducted with public health decision-makers, the needs and priorities of those primarily concerned with sexual health services (SHS) have not been fully considered. This research aimed to gain an insight into how decision-makers based in England, responsible for SHS use economic evidence to inform decision-making.

Methods In-depth semi-structured interviews with participants who were purposefully sampled through a snowballing approach until saturation was reached. The interviews were transcribed ad-verbatim and analysed using the framework analysis method.

Results Fifteen qualitative interviews with 17 participants were conducted. Eight participants were local commissioners, four were national commissioners, and three were responsible for service provision. Three main themes were identified:

Context/commissioning - Decision-making processes around SHS were reported as complex and involving multiple stakeholders. Different services are commissioned by a wide-range of decision-makers, and the different types of contracting SHS, affect the comparability of provision.

Costs and budgets - Decision-makers described pressures on budgets due to increasing demand for SHS and funding limitations. Nearly all stated that the fragmentation of commissioning created issues around budget flow. Another significant issue was that savings were realised by different parts of the system to those who pay for them.

Using economic evidence - Participants mainly focussed on economic evidence in terms of return on investment. Although broader outcomes such as impacts on inequalities were seen as relevant, this was often overshadowed by cost and cost-saving concerns. Helpful evidence was described as being adaptable to the local population and including costs relevant to local areas.

Conclusions Future economic evaluations of SHS need to be tailored to ensure that they provide economic evidence that meets the needs of decision-makers.

\section{P182 IDENTIFYING THE PREP GAP: A SYSTEMATIC REVIEW EXPLORING EQUITY IN THE HIV-PREP CARE CONTINUUM IN HIGH INCOME COUNTRIES}

${ }^{1} \mathrm{M}$ Cabecinha*, ${ }^{2} \mathrm{D}$ Solomon, ${ }^{1} \mathrm{G}$ Rait, ${ }^{3,4} \mathrm{~J}$ Saunders, ${ }^{4,5} \mathrm{H}$ Mohammed. ${ }^{1}$ Research Department of Primary Care and Population Health, University College London, London, UK ${ }^{2}$ Institute for Global Health, University College London, London, UK; ${ }^{3}$ UCL Centre for Clinical Research in Infection and Sexual Health, Institute for Global Health, University College London, London, UK; ${ }^{4}$ Blood Safety, Hepatitis, Sexually Transmitted Infections (STI) and HIV Division, National Infection Service, Public Health England, London, UK; ${ }^{5}$ Infection and Population Health, Institute for Global Health, University College London, London, UK

\subsection{6/sextrans-2021-sti.277}

Background Equitable implementation of HIV Pre-Exposure Prophylaxis (PrEP) is not well defined, particularly for populations already experiencing high levels of health inequity (e.g. people experiencing poverty or other social disadvantages). The five stages of the PrEP care-continuum (PCC) (awareness, acceptability, uptake, adherence, retention) can help evaluate PrEP implementation, but the extent to which key characteristics that are important for health equity are considered 